\title{
Dynamic Path-Following Using Temporary Path Generator for Mobile Robots with Nonholonomic Constraints
}

\author{
Seunghee Lee* \& Jong Hyeon Park ${ }^{* *}$ \\ Mcchatronics Lab., Precision Mechanical Eng., Hanyang Univ., \\ Seoul, South Korea \\ *1sh123@hymail.hanyang.ac.kr \\ **jongpark@email.hanyang.ac.kr
}

\begin{abstract}
The performance of dynamic path following of a wheeled mobile robot with nonholonomic constraints is highly affected by its initial states, because nonholonomically constrained system cannot guarantee the asymptotic stability for one equilibrium point of which errors and the time derivative of errors are zero. For this reason, the dynamic path following may yield poor performance with some initial states.

To overcome the initial state problems, we propose a temporary path generator, which generates a new temporary path with respect to the originally desired path. The direction of the path cannot be considered in dynamic path following, so we propose a new valid output, which can consider the dircction of a path. Simulation results are presented to demonstrate the effectiveness of the proposed algorithm of the dynamic path. From the simulation results, it is shown that the proposed algorithm has good performance and robustness to modeling errors.
\end{abstract}

\section{INTRODUCTION}

Whecled mobile robots arc typical examples of mechanical systems with nonholonomic constraints. But the dynamic control of mobile robots with nonholonomic constraints has been studied very recently. For nonholonomically constrained system, Bloch and McClamroch [9] first demonstrated that a nonholonomic system cannot be stabilized to a single equilibrium point of which errors and the time derivative of errors are zero, by a smooth feedback. They also showed that the system is small-time locally controllable. Campion et al. [10] showed that the system is controllable regardless of the structure of nonholonomic constraints. For nonholonomic mobile robots, Yamamoto [2] showed that the two wheeled nonholonomic mobile robots are not input-output linearizable for trajectory tracking of the center point of wheel axis as the output with static feedback, and presented the "Look-Ahead" output which is input-output linearizable.

Low level control of mobile platform can be broadly separated as two areas: path following $[7,8]$ and trajectory tracking $[2,5,3]$. In trajectory tracking problems, the output is a function of time explicitly, so the mobile robot must reach its desired point at the specified time, but not in the path following. In the problem of obstacle avoidance or following a complicated desired path, one may use the path following which has control performance that cannot be obtained from the trajectory tracking control for some specific case.

With the sense of trajectory tracking and path following, Sarkar, Yun, and Kumar [1] transformed the trajectory tracking problem to "dynamic" path following by selecting the output as the distance from the path and the ahead velocity of the platform. The dynamic path following has the advantage of the path following and the trajectory tracking, but there are some drawbacks in dynamic path following. In dynamic path following problem, the direction of the path cannot be considered, so the platform can follow the wrong direction. And for some states it is impossible to obtain the input torque. If the error of output is large, the computed input torque can be unrealistically large, and the systern can be unstable.

To overcome the above drawbacks of the dynamic path following and to improve the performance and stability of dynamic path following of mobile platform, we propose a temporary path generator and a new valid output. The system considered in this paper is nonsteerable and independently driven fixed two-wheeled mobile robot with nonholonomic constraints.

This paper is organized as follows. Section 2 presents the dynamic equation and input-output linearization of the wheeled mobile robot system. Section 3 presents the temporary path generator and the modified output for dynamic path following. In Section 4, simulations for 2 wheeled nonholonomic mobile platform are discussed. In Section 5, we present our conclusions.

\section{DYNAMIC PATH FOLLOWING}

We consider a wheeled mobile platform whose schematic top view is shown in Figure 1. If we ignore the passive wheels, the configuration of the platform can be described by four generalized coordinates: the wheel axis center position, $\left(x_{o}, y_{0}\right)$ and the angular position of the right and left wheels, $\theta_{r}$ and $\theta_{l}$. And thus

$$
\mathbf{q}=\left[\begin{array}{llll}
x_{o} & y_{o} & \theta_{r} & \theta_{l}
\end{array}\right]^{T}
$$

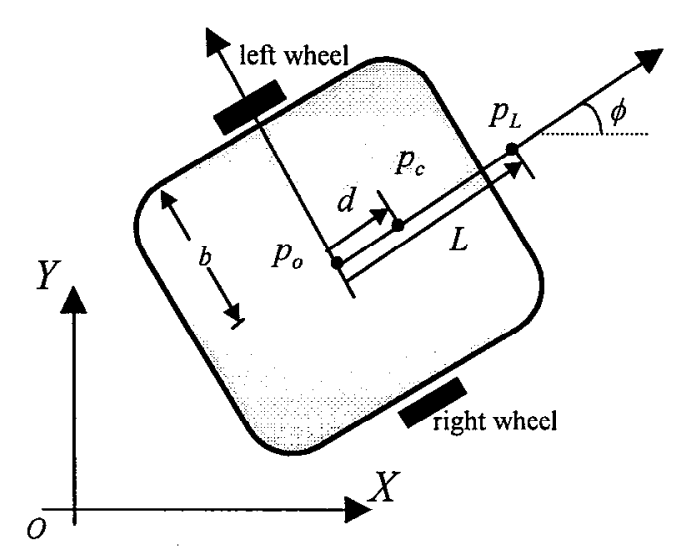

Fig.1. Schematic of the wheeled mobile platform. 
Under the Assumption that the driving wheels roll and do not slip, there are two nonholonomic constraints:

$$
\begin{gathered}
\dot{x}_{o} \sin \phi-\dot{y}_{o} \cos \phi=0 \\
\dot{x}_{o} \cos \phi+\dot{y}_{o} \sin \phi=c b\left(\theta_{r}+\theta_{l}\right),
\end{gathered}
$$

where $c=r / 2 b$ and $r$ is the radius of the wheel. The two nonholonomic constraints can be written in the form of

$$
A(\mathbf{q}) \dot{\mathbf{q}}=0 \text {, }
$$

where $A(\mathbf{q})=\left[\begin{array}{cccc}-\sin \phi & \cos \phi & 0 & 0 \\ -\cos \phi & \sin \phi & c b & c b\end{array}\right]$.

The dynamic equation of the system [2] is described by

$$
M(\mathbf{q}) \ddot{\mathbf{q}}+C(\mathbf{q}, \dot{\mathbf{q}})=E(\mathbf{q})-A^{T}(\mathbf{q}) \lambda,
$$

where $\lambda$ is the vectors of Lagrange multiplier for nonholonomic constraints.

If the system is subject to $k$ nonholonomic constraints, the number of generalized coordinates cannot be reduced by $k$. Therefore before well-known state space based control methods can be employed, an alternative approach is necessary to represent the motion and constraint equations in the state space. So to represent the dynamic equation as state-space form, let $S(\mathbf{q})$ be $4 \times 2$ matrix, whose columns are in the null space of $A(\mathbf{q})$, i.e., $A(\mathbf{q}) S(\mathbf{q})=0$. One of the possible $S(\mathbf{q})$ 's is

$$
S(\mathbf{q})=\left[\begin{array}{cc}
c b \cos \phi & c b \cos \phi \\
c b \sin \phi & c b \sin \phi \\
1 & 0 \\
0 & 1
\end{array}\right]
$$

From eq. (4), the velocity $\dot{\mathbf{q}}$ should be in the null space of $A(\mathbf{q})$. It follows that $\dot{\mathbf{q}} \in \operatorname{span}\left\{s_{1}(\mathbf{q}), s_{2}(\mathbf{q})\right\}$, and that there exists a smooth vector $\eta=\left[\begin{array}{ll}\eta_{1} & \eta_{2}\end{array}\right]^{T}$ such that

$$
\dot{\mathbf{q}}=S(\mathbf{q}) \eta
$$

Then

$$
\ddot{\mathbf{q}}=S(\mathbf{q}) \dot{\eta}+\dot{S}(\mathbf{q}) \eta
$$

For the specific choice as eq. (6) of $S(\mathbf{q}), \eta=\dot{\theta}$ where $\dot{\theta}=\left[\dot{\theta}_{r} \dot{\theta}_{l}\right]^{r}$.

Substitution eq. (8) into eq. (5),

$$
S^{T} M(S \dot{\eta}+\dot{S} \eta)+S^{T} C=\tau
$$

By choosing the state variable as

$$
\mathbf{x}=\left[\begin{array}{llllll}
x_{c} & y_{c} & \theta_{r} & \theta_{1} & \eta_{1} & \eta_{2}
\end{array}\right]^{T}
$$

Eq. (5) can be represented in the state space form of

$$
\dot{\mathbf{x}}=f(\mathbf{x})+g(\mathbf{x}) \tau
$$

$$
\text { where } \begin{aligned}
f(\mathbf{x}) & =\left[\begin{array}{c}
S \eta \\
-\left(S^{T} M S\right)^{-1}\left(S^{T} M \dot{S} \eta+S^{T} C\right)
\end{array}\right] \\
g(x) & =\left[\begin{array}{c}
0 \\
\left(S^{T} M S\right)^{-1}
\end{array}\right], \quad \eta=\left[\begin{array}{l}
\eta_{1} \\
\eta_{2}
\end{array}\right]=\left[\begin{array}{c}
\dot{\theta}_{r} \\
\dot{\theta}_{l}
\end{array}\right] .
\end{aligned}
$$

For simplification, suppose

$$
\begin{aligned}
\tau & =\alpha(\mathbf{x})+\beta(\mathbf{x}) \mu \\
& =\left(S^{T} M \dot{S} \eta+S^{T} C\right)+\left(S^{T} M S\right) \mu
\end{aligned}
$$

where $\mu$ is the new input variable. Then the closed-loop system becomes

where

$$
\dot{\mathbf{x}}=f^{1}(\mathbf{x})+g^{1}(\mathbf{x}) \mu
$$

$f^{1}(x)=\left[\begin{array}{c}S \eta \\ \mathbf{0}\end{array}\right], \quad g^{1}(\mathbf{x})=\left[\begin{array}{c}\mathbf{0} \\ I_{2}\end{array}\right]$.

If we analyze automobile maneuvering, the two most important requirements are to follow the road by staying as close to the path as possible and to maintain the desired forward velocity. So, in dynamic path following, the output can be chosen as the distance from the path and the ahead velocity of the platform.

Dubins [6] and later Reeds and Shepp [7] showed that given any initial and final position and orientation of a platform, there exists a family of paths composed of only straight line and circular arc segments between any two points. In fact, Dubins proved that this family contains the R-geodesic between the two points. Sarkar et al. [1] use this result to suppose that any path can be suitably broken down into straight line and circular arc segments. Therefore if it is possible to control the mobile platform on such basic paths composed of the basic segments, it can effectively move itself from any position and orientation to any other position and orientation.

Let's consider a circular path. Let $P_{f}$ be the center of the circular path and $\left(x_{f}, y_{f}\right)$ be its position in the world coordinate system. Let $R$ be the constant radius of the circular path. One output chosen here is

$$
y_{1}=\sqrt{\left(x_{L}-x_{f}\right)^{2}+\left(y_{L}-y_{f}\right)^{2}}-R,
$$

where $\left(x_{L}, y_{L}\right)$ is the location of the look-ahead point shown in Figure 1. Thus,

$$
\begin{aligned}
& x_{L}=x_{o}+L \cos \phi \\
& y_{L}=y_{c}+L \sin \phi
\end{aligned}
$$

Note that the shortest distance from point $P_{L}$ to the circular path is absolute value of $y_{1}$.

Note that the shortest distance from point $P_{L}$ to the circular path is the absolute value of $y_{1}$.

The forward velocity of the platform chosen as the other output is

$$
y_{2}=\dot{x}_{o} \cos \phi+\dot{y}_{o} \sin \phi=\frac{r}{2}\left(\eta_{1}+\eta_{2}\right)
$$


Differentiating eqs. (14) and (17),

$$
\begin{aligned}
& \dot{y}_{1}=\frac{\partial y_{1}}{\partial \mathbf{x}} \dot{\mathbf{x}}=J_{h 1}(\mathbf{q}) S(\mathbf{q}) \eta \\
& \ddot{y}_{1}=\frac{d}{d t}\left(J_{h 1} S\right) \eta+J_{h 1} S \mu=u_{1} \\
& \dot{y}_{2}=J_{h 2} \mu=u_{2}
\end{aligned}
$$

where

$$
\begin{aligned}
& J_{h 1}=\frac{\partial h_{1}}{\partial \mathbf{q}}=\frac{1}{d_{k}}\left[\begin{array}{c}
x_{L}-x_{f} \\
y_{L}-y_{f} \\
-L c\left(x_{o}-x_{f}\right) \sin \phi+L c\left(y_{o}-y_{f}\right) \cos \phi \\
L c\left(x_{o}-x_{f}\right) \sin \phi-L c\left(y_{o}-y_{f}\right) \cos \phi
\end{array}\right]^{T}, \\
& J_{h 2}=\left[\begin{array}{ll}
\frac{r}{2} & \frac{r}{2}
\end{array}\right], d_{k}=\sqrt{\left(x_{L}-x_{f}\right)^{2}+\left(y_{L}-y_{f}\right)^{2}}, \\
& u_{1}=-k_{1} \dot{y}_{1}-k_{2} y_{1}, \\
& u_{2}=\dot{y}_{2 d}-k_{3}\left(y_{2}-y_{2 d}\right), \\
& k_{1}, k_{2} \text {, and } k_{3} \text { are linear control gains. }
\end{aligned}
$$

Then eqs. (18), (19), and (20) can be represented by

$$
\Phi \mu=\left[\begin{array}{c}
u_{1}-\frac{d}{d t}\left(J_{h 1} S\right) \eta \\
u_{2}
\end{array}\right]
$$

where the decoupling matrix $\Phi$ is $\Phi=\left[\begin{array}{c}J_{h 1} S \\ J_{h 2}\end{array}\right]$.

The determinant of $\Phi$ is computed as

$$
\operatorname{det}(\Phi)=\frac{r c(d+L)}{d_{k}}\left(\left(y_{o}-y_{f}\right) \cos \phi-\left(x_{o}-x_{f}\right) \sin \phi\right)
$$

Although $y_{1}$ in this case is not the true distance, it is a measure of the closeness of the mobile robot to the given path. The reason of using look-ahead point $\left(x_{L}, y_{L}\right)$ instead of the wheel axis center point $\left(x_{o}, y_{o}\right)$ is that using the wheel axis center point doesn't lead the system to be input-output linearizable in static feedback. If the output equations are chosen as

$$
\begin{aligned}
& y_{1}=\sqrt{\left(x_{c}-x_{f}\right)^{2}+\left(y_{c}-y_{f}\right)^{2}}-R \\
& y_{2}=\frac{r}{2}\left(\eta_{1}+\eta_{2}\right),
\end{aligned}
$$

$\Phi$ remains singular for all states.

For a straight line path described by $A x+B y+C=0$, the output equations are chosen as

$$
\begin{aligned}
& y_{1}=\frac{A x_{L}+B y_{L}+C}{\sqrt{A^{2}+B^{2}}} \\
& y_{2}=\frac{r}{2}\left(\eta_{1}+\eta_{2}\right),
\end{aligned}
$$

where

$$
J_{h 1}=\frac{1}{\sqrt{A^{2}+B^{2}}}\left[\begin{array}{c}
A \\
B \\
-A L \sin \phi+B L \cos \phi \\
A L \sin \phi-B L \cos \phi
\end{array}\right]^{T} \quad J_{h 2}=\left[\begin{array}{ll}
\frac{r}{2} & \frac{r}{2}
\end{array}\right] .
$$

\section{TEMPORARY PATH GENERATOR}

The output equations for the dynamic path following proposed by Sarkar et al. [1] have the following three drawbacks.

1) When $\Phi$ is singular, the input cannot be calculated. Eq. (22) indicates that $\Phi$ is singular when

$$
\left(x_{L}-x_{f}\right) \sin \phi-\left(y_{L}-y_{f}\right) \cos \phi=0 \text { for the circular path, }
$$

and

$$
B \cos \phi-A \sin \phi=0 \text { for the straight line path, }
$$

which means that $\Phi$ is singular when the heading direction of the platform is normal to the circular path, and when the heading direction of the platform is normal to the straight line. If the error in the position and the velocity are large, such a singular condition is apt to occur.

2) The direction of the path cannot be considered. If the platform must follow a complicated path, or the initial direction of the platform is close to opposite direction with the path direction, the platform cannot follow the correct direction in dynamic path following.

3) If the error of distance to path and the desired velocity are large, the calculated input torque can be unrealistically large, and the system can be unstable.

To overcome the drawbacks of the outputs in Section 2, we propose a temporary path generator and a modified output. If the temporary path generator is used in the dynamic path following, the error of distance to path is limited to small value, so the calculated input torque cannot be unrealistically large value when the desired velocity is not very large.

Since a path is decomposed into circles and straight lines, cases depending on the relative position of the platform to the path:

1) when the position of platform is out of the circle path

2) when the position of platform is inside of circle path

3) when the position of platform is out of the straight line.

Circular temporary path of radius $R_{t}$ is generated

Case 1)

$R_{t}=y_{1}+\zeta R$ when the position error is larger than $R / 2$
,where $\zeta$ is a constant.

$R_{t}=R$ otherwise

Case 2)

when the position of the platform is not $P_{f}$

$$
R_{t}=\frac{\left(R+\sqrt{\left(x_{L}-x_{f}\right)^{2}+\left(y_{L}-y_{f}\right)^{2}}\right)}{2}-\zeta R
$$

$$
R_{t}=R / 2 \quad \text { otherwise. }
$$

Case 3)

where $R_{o}$ is a constant. 
Since there exist 2 circular temporary generated at a particular situation, as shown in Figure 2, an appropriate one should be selected with respect to the desired path direction.

Although with the temporary path, in order to overcome the direction and singular problem, the following modified output for the velocity term is proposed.

$$
y_{2}=a_{1} \frac{r}{2}\left(\eta_{1}+\eta_{2}\right)+a_{2}\left(1-a_{1}\right)\left(\eta_{1}-\eta_{2}\right)
$$

Where $a_{1}$ is determined by the difference between the path direction and the platform direction as shown in Figure 3.

$$
a_{1}=\frac{1}{2}(1+\vec{p} \cdot \vec{d})
$$

and variable $a_{2}$ is defined as

$$
a_{2}=\operatorname{sign}((\vec{p} \times \vec{d}) \cdot \hat{k}),
$$

which describes the direction in which the platform has to move.

Then,

$$
J_{h 2}=\left[\frac{a_{1} r}{2}+a_{2}\left(1-a_{1}\right) c L \quad \frac{a_{1} r}{2}-a_{2}\left(1-a_{1}\right) c L\right]
$$

Eq. (30) can be represented by

$$
a_{1}=\frac{1}{2} \frac{d_{k}-\left(x_{L}-x_{f}\right) \sin \phi+\left(y_{L}-y_{f}\right) \cos \phi}{d_{k}}
$$

or

$$
a_{1}=\frac{1}{2} \frac{d_{k}+\left(x_{L}-x_{f}\right) \sin \phi-\left(y_{L}-y_{f}\right) \cos \phi}{d_{k}}
$$

The singular condition of the corresponding $\Phi$ becomes

$$
a_{1}=\frac{\alpha}{\alpha+\beta},
$$

where

$$
\begin{aligned}
& \alpha=\left(x_{c}-x_{f}\right) \cos \phi+\left(y_{c}-y_{f}\right) \sin \phi+L \\
& \beta=\left(y_{c}-y_{f}\right) \cos \phi-\left(x_{c}-x_{f}\right) \sin \phi
\end{aligned}
$$

By the calculation of $a_{1}$ in eqs. (33), (34), and (35), the singular condition of $\Phi$ is as follows when $a_{2}=1$

$$
\frac{\alpha}{\alpha+\beta}=\frac{1}{2}\left(\frac{d_{k}+\beta}{d_{k}}\right)
$$

or

$$
\beta^{2}+\left(d_{k}+\alpha\right) \beta-d_{k} \alpha=0
$$

We check the singularity for all case of $\alpha$ and $\beta$.

Case 1. $\beta>0, \alpha<0$

$$
\frac{\alpha}{\alpha+\beta}<0 \text { or } \frac{\alpha}{\alpha+\beta}>1 \therefore \text { non-singular }
$$

Case 2. $\beta>0, \alpha=0$

$$
\frac{\alpha}{\alpha+\beta}=0 \text { and } a_{1}=\frac{1}{2}\left(\frac{d_{k}+\beta}{d_{k}}\right)>\frac{1}{2} \therefore \text { non-singular }
$$

Case 3. $\beta=0, \alpha=0$

$$
\frac{\alpha}{\alpha+\beta}=1 \text { and } a_{1}=\frac{1}{2}\left(\frac{d_{k}+\beta}{d_{k}}\right)=\frac{1}{2} \quad \therefore \text { non-singular }
$$

Case 4. $\beta<0, \alpha<0$

$$
\begin{aligned}
\beta^{2}+\left(d_{k}+\alpha\right) \beta-d_{k} \alpha & >\beta^{2}+\left(d_{k}+\alpha\right) \beta \\
& =\beta\left(\beta+d_{k}+\alpha\right)>0
\end{aligned} \therefore \text { non-singular }
$$

Case 5. $\beta<0, \alpha=0$

$$
a_{1}=\frac{d_{k}+\beta}{d_{k}} \neq \frac{\alpha}{\alpha+\beta} \text { only if } \beta \neq-d_{k}
$$

In order to avoid singularity, the lower bound of $a_{1}$ must be positive.

When $a_{2}=-1$, the result has the same trend with above result. With the modified output equation, the path direction can be also considered. Moreover the decoupling matrix $\Phi$ remains nonsingular all the time.

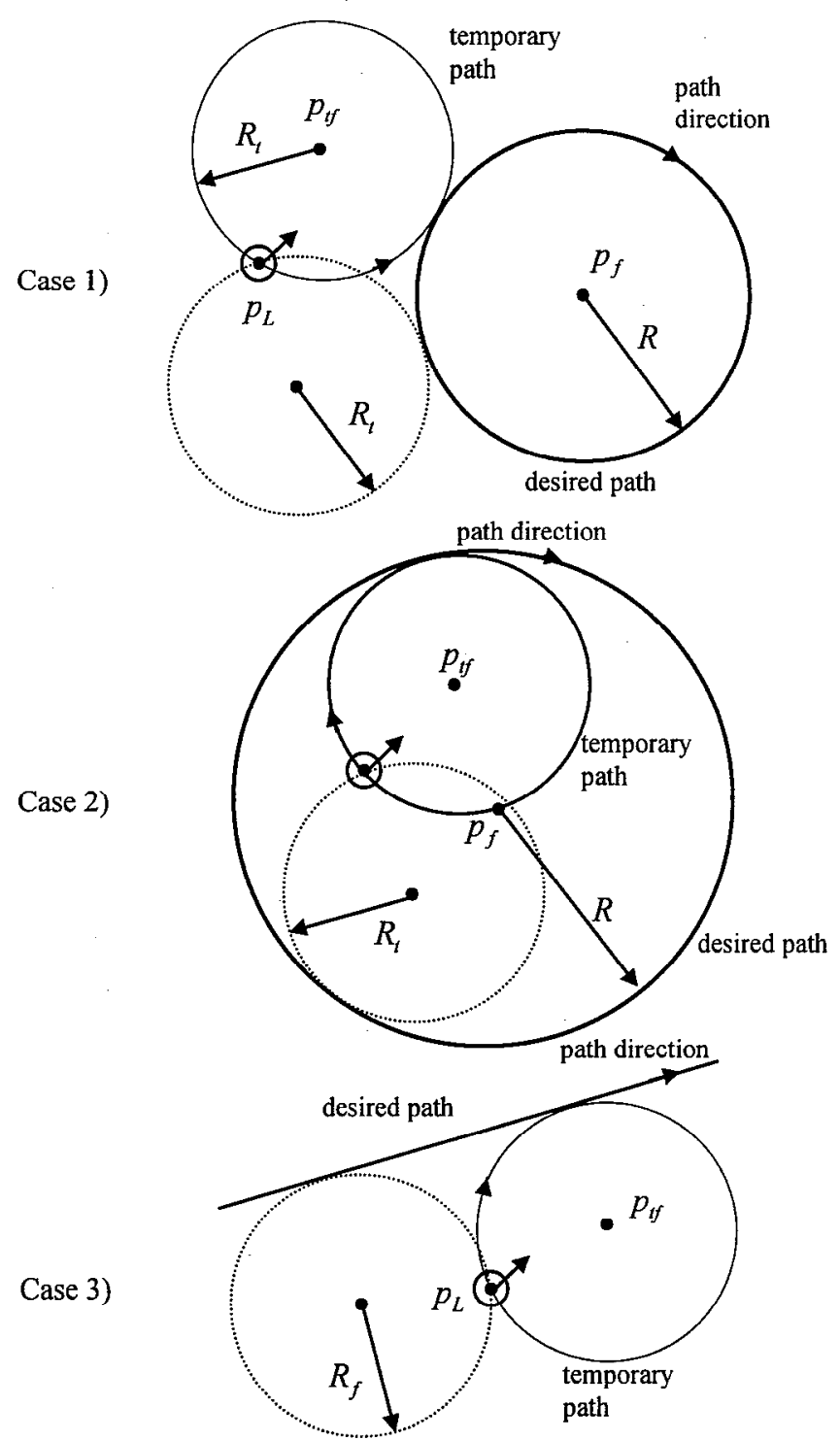

Fig.2 Temporary path generation for the case 1),2) and 3) 
path direction

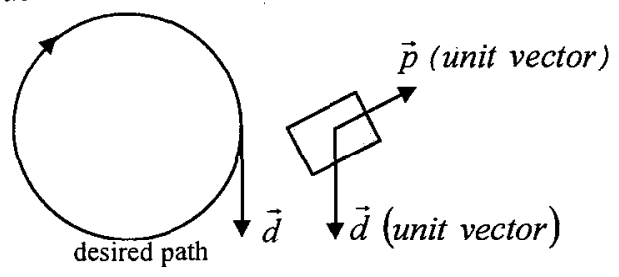

Fig. 3. Determination of $a_{1}$ and $a_{2}$

To characterize the zcro dynamics, a new state space variable $z$ defined as follows.

$$
z=T(\mathbf{x})=\left[\begin{array}{c}
z_{1} \\
z_{2} \\
z_{3} \\
z_{4} \\
z_{5} \\
z_{6}
\end{array}\right]=\left[\begin{array}{c}
y_{1} \\
y_{2} \\
\dot{y}_{1} \\
\theta_{r} \\
\theta_{l} \\
\tan ^{-1}\left(\frac{x_{c}-x_{f}}{y_{c}-y_{f}}\right)
\end{array}\right],
$$

where $T(\mathbf{x})$ is a diffeomorphism [4].

Input-output linearization as well as input-output decoupling is possible by noting the observable part of the system.

After some tedious calculations, it can be shown that the zero dynamics of the system is

$$
\dot{z}_{3}=\dot{z}_{4}=\dot{z}_{5}=0
$$

which is clearly Lagrange stable but not asymptotically stable.

\section{SIMULATION}

Computer simulations were performed to show the effectiveness of the temporary path generator and modified output. The platform and control parameters used in the simulations are summarized in Table 1. Figure 4 shows a case when a singular condition of decoupling matrix $\Phi$ occurs and the simulation cannot be continued. When the temporary path generator is used, the platform follows its path correctly, and the error in the velocity converges to zero, as shown in Figure 6 . The input torque is reasonably small as in Figure 7 . Without the modified output, the platform cannot follow the correct direction as in Figure 8, while with the modified output it follows the correct direction though the path and the platform has a reverse direction with each other. Even if the platform inside of the circle, the platform follows the path correctly as in Figure 10. Figure 11 shows that the controller which we propose has the robustness for the modeling error.

\section{CONCLUSION}

The temporary path generator that we propose can guarantee the local stability of the system without excessive input torque. And even in the case of breaking away from the desired path, the generator makes a temporary path so that the system can return to the desired path quickly. Even when the initial direction of the platform is different from that of the desired path, it is possible to follow the right direction of the path with the modified output proposed here. With proposed method, errors in the position and velocity converge to zero fast. The simulation results show that the proposed path generator and modified output can overcome the initial condition problem and path direction problem, and that the controller has the robustness to modeling errors.

\section{REFERENCES}

[1] N. Sarkar, X. Yun, and U. Kumar, "Control of Mechanical Systems with Rolling Constraints: Application to Dynamic Control of Mobile Robots", Int. J. of Robotics Research, vol. 13, no.1, 1994.

[2] Y. Yamamoto, "Control and Coordination of Locomotion and Manipulation of a Wheeled Mobile Manipulators", Ph. D. thesis, University of Pennsylvania, 1994.

[3] C. C. de Wit, B. Siciliano, and G. Bastin, "Theory of Robot Control", pp. 265-357, Springer, 1996.

[4] J.-J. E. Slotine, "Applied Nonlinear Control", pp. 207-275, Prentice-Hall international editions, 1991.

[5] J. Yang, I. Choi, and J. Kim, "Sliding Mode control of a Nonholonomic wheeled Mobile Robot for Trajectory Tracking", Proc. Int. Conf. on Robotics and Automation, pp. 1983-1988, Leuven, Belgium, May 1998.

[6] L. E. Dubins, "On Curves of Minimal Length with a Constraint on Average Curvature, and with Prescribed Initial and Terminal Positions and Tangents", American Journal of Mathematics, vol. 79, pp. 497-516, 1957.

[7] J.A. Reeds and L.A. Shepp, "Optimal Paths for a Car that goes Both Forwards and Backwards.", Pacific Journal of Mathematics, vol.145, no.2, pp.367-393, 1990.

[8] C. C. de Wit and R. Roskam, "Path Following of a 2-DOF Wheeled Mobile Robot Under Path and Input Torque Constraints.", Proc. Int. Conf. on Robotics and Automation, pp. 1142-1147, Sacramento, CA, April 1991.

[9] A. Bolch and N. H. McClamroch, "Control of mechanical systems with classical nonholonomic constraints.", Proc. of $28^{\text {th }}$ IEEE Conf. on Decision and Control, pp.201-205, Tampa, FL, December 1989.

[10] G. Campion, B. d'Andrea Novel, and G. Bastin, "Controllability and state feedback stabilization of nonholonomic mechanical systems.", In C. Canudas de Wit, editor, Lecture Notes in control and Information Science, volume 162, pp. 106-224. Springer-Verlag, 1991. 


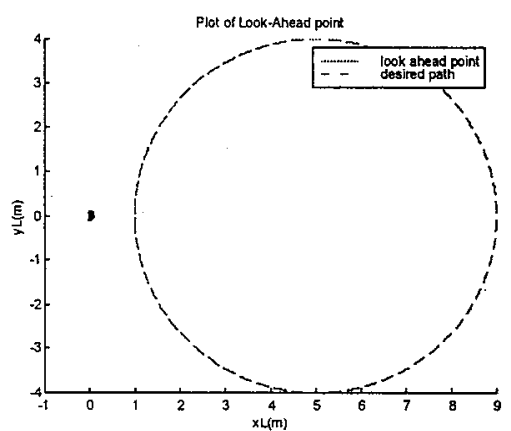

Fig.4. Dynamic path following without the temporary path generator when desired velocity of the platform is $1 \mathrm{~m} / \mathrm{s}$

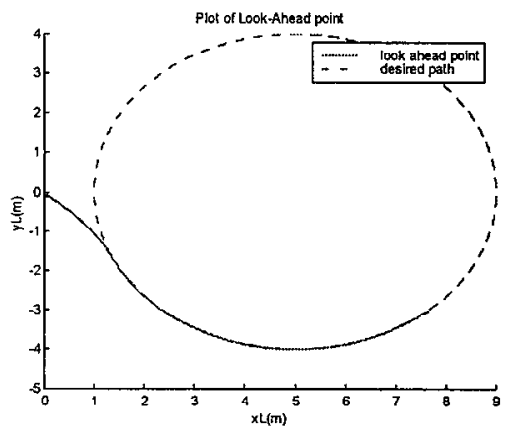

Fig. 5. Dynamic path following with the temporary path generator and modified output when desired velocity of the platform is $1 \mathrm{~m} / \mathrm{s}$

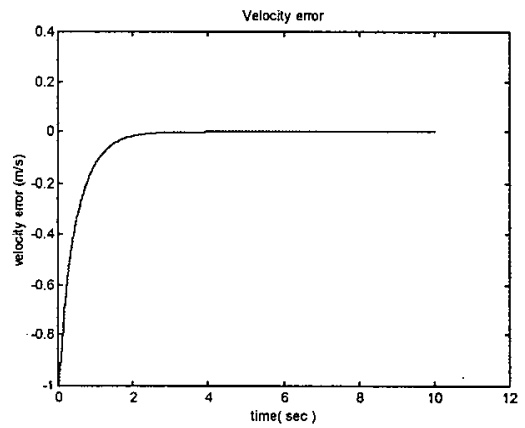

Fig. 6. Velocity error of the dynamic path following with the temporary path generator and modified output.

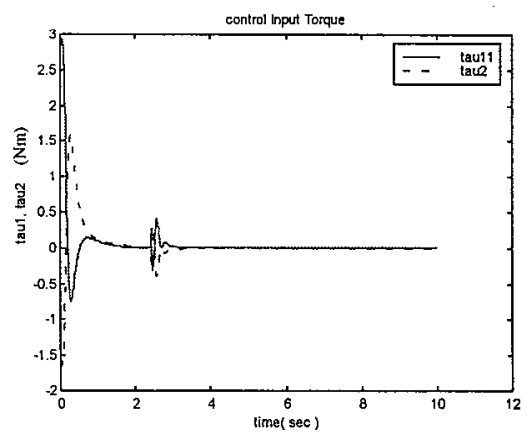

Fig. 7. Calculated input torque of the dynamic path following with the temporary path generator and modified output.

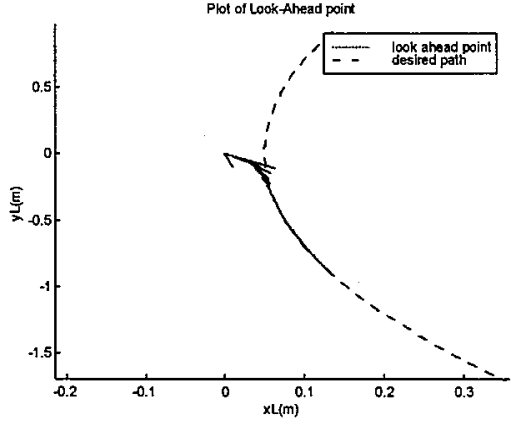

Fig.8. Dynamic path following without the temporary path generator when the desired path direction is CW.

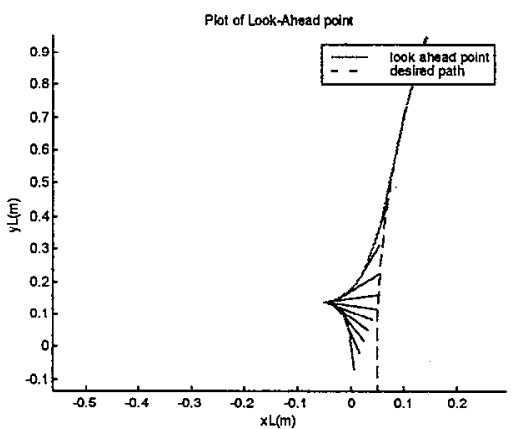

Fig. 9. Dynamic path following with the temporary path generator and modified output when the desired path direction is $\mathrm{CW}$.

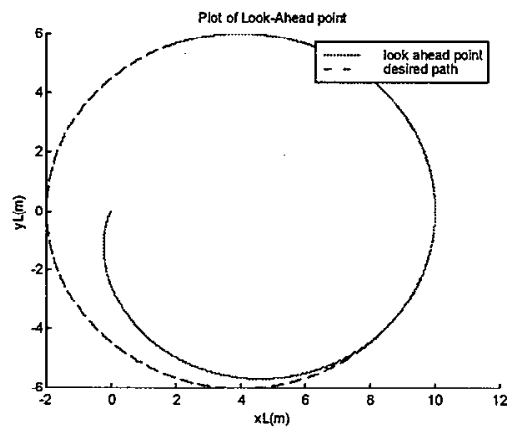

Fig. 10. Dynamic path following with the temporary path generator and modified output when the desired path direction is CW.

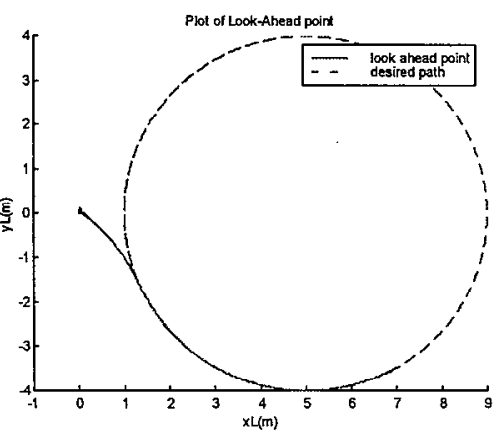

Fig.11. Dynamic path following with the temporary path generator and modified output when the total mass and mass moment of inertia are increased by $300 \%$.

Table 1. The parameters of the simulation

\begin{tabular}{|c|c|c|c|c|c|c|c|c|}
\hline $\begin{array}{c}\text { total } \\
\text { mass }\end{array}$ & $\mathrm{r}$ & $\mathrm{b}$ & $\mathrm{L}$ & $\begin{array}{c}\text { rotation } \\
\text { speed }\end{array}$ & $\begin{array}{c}\text { temporary } \\
\text { path margin }\end{array}$ & $k_{1}$ & $k_{2}$ & $k_{3}$ \\
\hline $10.6 \mathrm{Kg}$ & $0.08 \mathrm{~m}$ & $0.3 \mathrm{~m}$ & 0.08 & $0.1 \mathrm{~m} / \mathrm{s}$ & $0.01 \mathrm{~m}$ & 40 & 400 & 4 \\
\hline
\end{tabular}

